\title{
AMEGO: Simulations of the Instrument performance (ICRC2017)
}

\author{
Regina Caputo* \\ UMD/NASA/GSFC \\ E-mail: regina.caputo@nasa.gov
}

\section{Fabian Kislat ${ }^{a}$, Judith Racusin ${ }^{b}$}

${ }^{a}$ Department of Physics and McDonnell Center for the Space Sciences, Washington University in St. Louis, Saint Louis, MO 63130, USA

${ }^{b} N A S A / G S F C$

\section{The AMEGO Team}

https://asd.gsfc.nasa.gov/amego/

\begin{abstract}
The gamma-ray energy range from several hundred keV to a hundred $\mathrm{MeV}$ has remained largely unexplored since the observations by instruments on the Compton Gamma-Ray Observatory (1991- 2000) and on INTEGRAL (since 2002). This energy range is particularly challenging because it is firmly in the Compton-dominated regime where the interaction cross section is minimized. Accurate measurements are critical for answering a broad range of astrophysical questions. To address these questions, we are developing AMEGO: All-sky Medium Energy Gammaray Observatory, to investigate the energy range from $200 \mathrm{keV}$ to $>10 \mathrm{GeV}$ with good energy and angular resolution and with sensitivity approaching a factor of 20-50 better than previous measurements. This instrument will be capable of measuring both Compton-scattering events at lower energies and pair-production events at higher energies. To achieve these ambitions goals Monte Carlo (MC) simulations will play a crucial role guiding the design of AMEGO. I will present an overview of the AMEGO simulation campaign using the MEGAlib framework, as well as the initial results for effective area and angular resolution, as well as sensitivity projections.
\end{abstract}

35th International Cosmic Ray Conference - ICRC2017

10-20 July, 2017

Bexco, Busan, Korea

${ }^{*}$ Speaker. 


\section{AMEGO}

The All-Sky Medium Energy Gamma-ray Observatory (AMEGO) is a mission in consideration as a probe for the 2020 Astrophysics Decadal review. AMEGO will survey the entire sky every 3 hours and see $80 \%$ of the sky every orbit with its wide field of view $(\sim 2.5 \mathrm{sr})$ and excellent continuum sensitivity between $200 \mathrm{keV}$ and $10 \mathrm{GeV}$. The AMEGO design primarily uses well understood, well tested technologies with significant space heritage.

AMEGO will detect medium energy gamma-rays via pair production (between $\sim 10 \mathrm{MeV}$ and $10 \mathrm{GeV}$ ) and via Compton Scattering (between $200 \mathrm{keV}$ and $\sim 10 \mathrm{MeV}$ ). An incoming photon will undergo one of these interactions in a tracker composed of 60 layers of double sided silicon strip detectors coupled to an analogue readout. This tracker will record the energies and tracks of electrons and positrons as they pass through it. A Cadmium Zinc Telluride (CZT) calorimeter is placed under the tracker as well as half way up the sides to measure the location and energy of Compton scattered photons. Finally a CsI calorimeter resides under the lower CZT which extends the upper energy range by measuring the energies of the particles produced via pair production and provides sensitivity to polarization and increased spectral capabilities. The full instrument is surrounded by a plastic anti coincidence detector to veto charge particle backgrounds. Figure 1 is a diagram of the instrument [1].

\section{Science Goals}

The AMEGO energy band is the transition between the thermal and non-thermal Universe. It is the only part of the electromagnetic spectrum where it is possible to directly observe nuclear processes (atomic nuclei de-excitations and excitations). Specifically, it covers the positron annihilation line at $511 \mathrm{keV}$. Also, large populations of known sources exist with their peak power output in the $\mathrm{MeV}$ range making it crucial to study for source energetics. The AMEGO mission will initiate breakthroughs in our understanding of extreme environments. These physical properties exist in an array of objects including pulsars and magnetars, $\gamma$-ray bursts and multi-messenger astrophysics [2], active galaxies [3], and dark matter [4].

\section{Simulation Overview}

To fully realize the capabilities of AMEGO, we perform detailed instrument simulations using the MEGAlib toolkit [5]. Simulations and event reconstruction are performed using the MEGAlib framework which requires ROOT (v6) and Geant4 (v10). The detector volume is built in Geomega (Geometry for MEGAlib). The geometry file describes the volume, the materials, the detectors, the trigger criteria. It is used by all programs in the MEGAlib framework including the Geant4 simulation. The event simulation is done using Cosima (COsmic SImulator for MEGAlib based on Geant4) [6]. For our studies of the performance we have used a 'FarFieldPointSource', unless otherwise specified, in which particles are emitted from a disk defined by a surrounding sphere. Events are generated as a function of energy and incidence angle $(\cos (\theta))$ with 100k triggers.

Event reconstruction is done using Revan (Real EVent ANalyzer) [7]. It utilizes the simulation files which are output from Cosima. Revan reconstructs Compton scattering, pair conversion and 
charged particles (muons, protons, etc). These are referred to as "event types". The algorithm first reconstructs tracks and searches for vertices in those tracks from pair conversion. If a vertex is not found, the tracks are checked for consistencies with charged particles (hits in the ACD, etc). If these criteria are not met, events are sent to the Compton reconstruction algorithm. Compton events are further divided into events in which the scattered electron is recovered (tracked) and events in which the scattered electron is not (untracked). Untracked events are particularly challenging because only the scattered photon is measured; however, this event class is most important for the lowest energy events. For these studies, we consider only tracked Compton events, leaving untracked Compton events for future studies. In the rest of this document, "Compton events" will refer to tracked Compton events only. For further details, please see Ref. [7]. Revan is optimized for Compton events. One main outcome of this study is that a new pair conversion algorithm (based of the Fermi-LAT event reconstruction) will be written.

For the main data analysis, we have developed a new program, EventAnalysis, which uses the reconstructed events from Revan. We validated this program with the MEGAlib algorithm Mimrec. With EventAnalysis, we make additional event selections. Specifically we include a $60^{\circ}$ cut on the opening angle of pair conversion events. We accept any event that is reconstructed with an ARM of $10^{\circ}$ assuming that with more detailed event reconstruction for a flight instrument, we would be able to recover more events. The AMEGO geometry and simulation files can be found on GitHub. ${ }^{1}$

\section{AMEGO Performance}

The main parameters which determine the performance of the instrument are the angular resolution (ARM), the Effective Area $\left(A_{\text {eff }}\right)$, and the $3 \sigma$ source sensitivity. The sensitivity requires the ARM and the $A_{\text {eff }}$.

\subsection{Angular Resolution}

We start by simulating the ARM of the AMEGO instrument (based on the true generated events). The ARM is defined as the difference between the reconstructed and true direction in the following ways for the different event types:

\footnotetext{
${ }^{1}$ https://github.com/ComPair
}

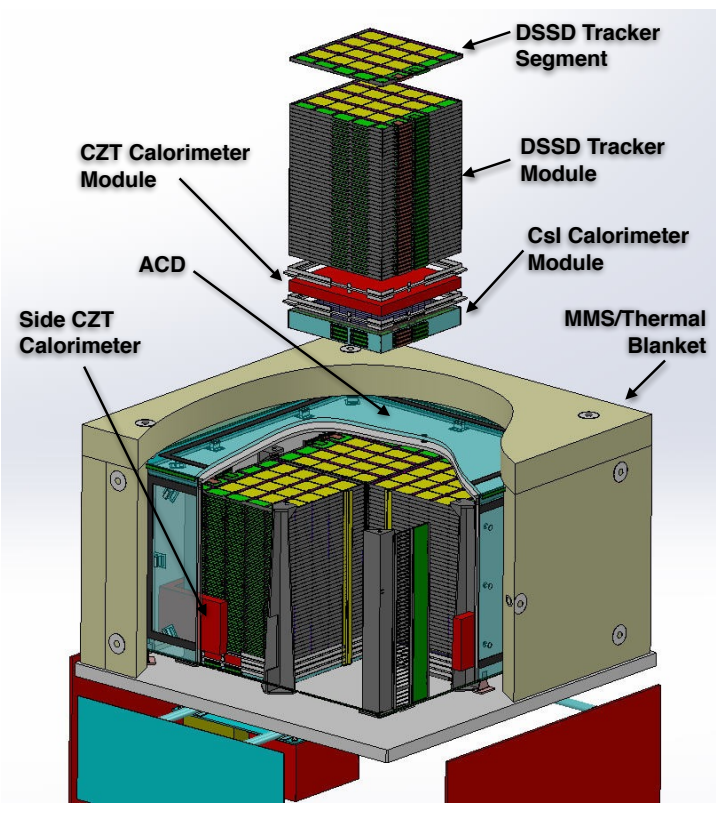

Figure 1: The AMEGO hardware design [1]. 
- ARM Compton Events: The angular resolution is the angular distance from the true known direction to the outer edge of the Compton cone (or arc).

- ARM Pair Events: The reconstructed direction is determined by the bisect angle of the electron and positron direction vectors, weighted by energy. The ARM is the $68 \%$ containment of the resulting distribution.

Details on the calculations of the ARM can be found in Ref. [7].

We report the ARM for the different events types as a function of energy for two different incident angles in Fig. 2 (left). We also report the ARM as a function of incident angle for two different energies (one firmly in the pair-production regime and one in the Compton regime) in Fig. 2 (right). It is worth noting that the most challenging regime is in the middle of our energy band: $\sim 10 \mathrm{MeV}$. This energy is the transition region between the Compton scattering and pairproduction cross sections.
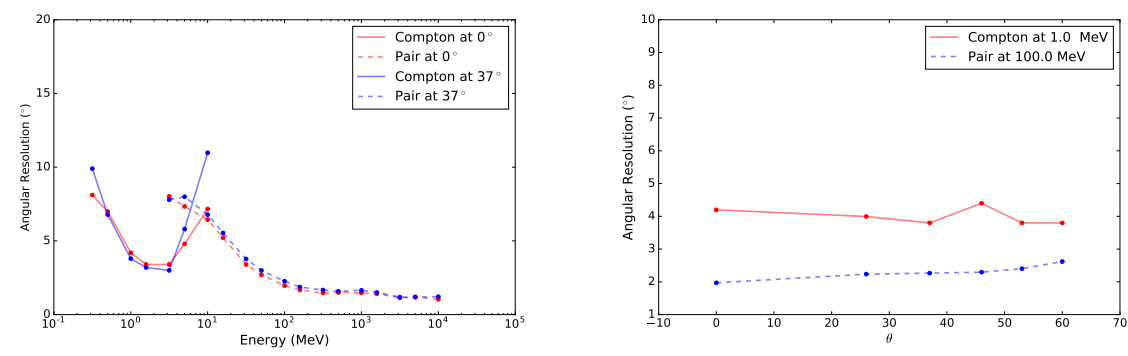

Figure 2: Angular resolution as a function of true incident energy for two incident angles $\left(0^{\circ}\right.$ and $\left.37^{\circ}\right)($ left $)$ and as a function of the true incident angle for two different energies ( $1 \mathrm{MeV}$ and $100 \mathrm{MeV})$ (right) for two event types. The two energies were chosen to represent events firmly in the Compton and pair-production regimes respectively.

\subsection{Effective Area}

We also determine the $A_{\text {eff }}$ of the AMEGO instrument. This was done using the relationship:

$$
A_{\text {eff }}=\frac{N_{R}}{N_{T}} \times A_{\text {sphere }}
$$

15

\section{Sensitivity Curves}

Once we determined the the ARM and $A_{\text {eff }}$ for the AMEGO instrument, we calculated the expected source sensitivity. The sensitivity is dependent on the background model which is shown in Fig. 4. For this initial study we include astrophysical backgrounds (galactic), extragalactic backgrounds and diffuse emission, discussed in greater detail in Sec. 5.1. 

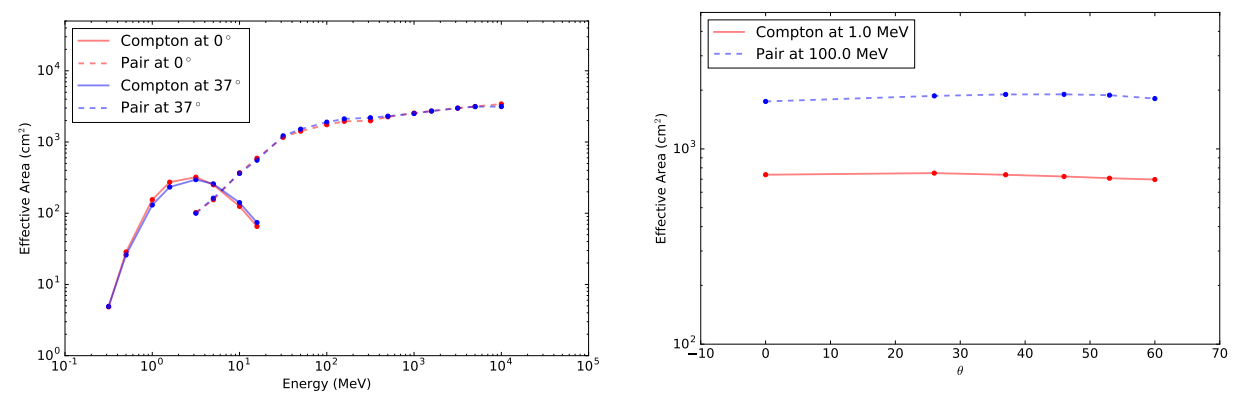

Figure 3: Effective Area $\left(\mathrm{A}_{\text {eff }}\right)$ as a function of true incident energy for two incident angles $\left(0^{\circ}\right.$ and $\left.37^{\circ}\right)($ left $)$ and as a function of the true incident angle for two different energies ( $1 \mathrm{MeV}$ and $100 \mathrm{MeV}$ ) (right) for two event types. The two energies were chosen to represent events firmly in the Compton and pair-production regimes respectively.

\subsection{Background Description}

The background spectrum in the AMEGO energy range is composed of cosmic photon sources (Galactic and Isotropic), charged particles from cosmic sources and the Earth's Albedo, atmospheric secondary gamma rays, internal instrument background (from the activation of the CsI 5 calorimeter), and the charged particles from exiting and entering the South Atlantic Anomaly (SAA).

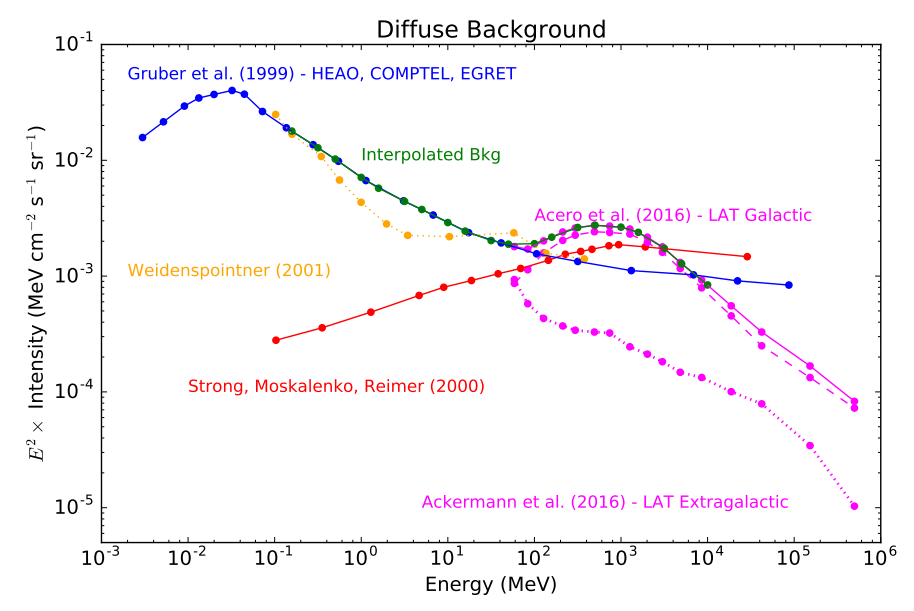

Figure 4: Measurements of cosmic background in the AMEGO energy range. Backgrounds used in these simulations are [8] and [9] (high Galactic latitude), with a conservative (x10) scaling factor in the Compton regime. Additional background measurements for comparison include Refs. [10, 11, 12].

A literature search for background measurements in this energy range yielded a variety of measurements as summarized in Figure 4. Most of the measurements were in rough agreement, so we chose to use the most conservative estimates measured with HEAO, COMPTEL, and EGRET [8] in the Compton regime, and measurements from Fermi-LAT in the pair regime [9].

Our preliminary simulations included only cosmic photon sources with a conservative scaling factor (x10) applied to Compton regime to account for the unknown Albedo component. Since 
these backgrounds are better understood in the pair regime, no additional scaling factor was used. Current studies are on-going to more accurately account for these other components; however, initial results show that the scale factor of 10 is a reasonably conservative estimate.

\subsection{Sensitivity}

We calculated the sensitivity using the following formula:

$$
I_{\mathrm{src}}=\frac{E}{A_{\mathrm{eff}} T_{\mathrm{obs}}} \times\left(\frac{n_{\mathrm{sig}}^{2}}{2}+\sqrt{\frac{n_{\mathrm{sig}}^{4}}{4}+\frac{n_{\mathrm{sig}}^{2} F_{\mathrm{bkg}} A_{\mathrm{eff}} T_{\mathrm{obs}} d \Omega}{E}}\right)
$$

where $E$ is the energy, $A_{\text {eff }}$ is the effective area, $n_{\text {sig }}$ is the significance, $F_{\mathrm{bkg}}$ is the background flux in $\mathrm{MeV} /\left(\mathrm{cm}^{2} \mathrm{~s} \mathrm{sr}\right)$, and $T_{\text {obs }}$ is the observation time. $d \Omega$ is defined here (where the ARM is the angular resolution):

$$
d \Omega=2 \pi(1-\cos (2 \times \mathrm{ARM}))
$$

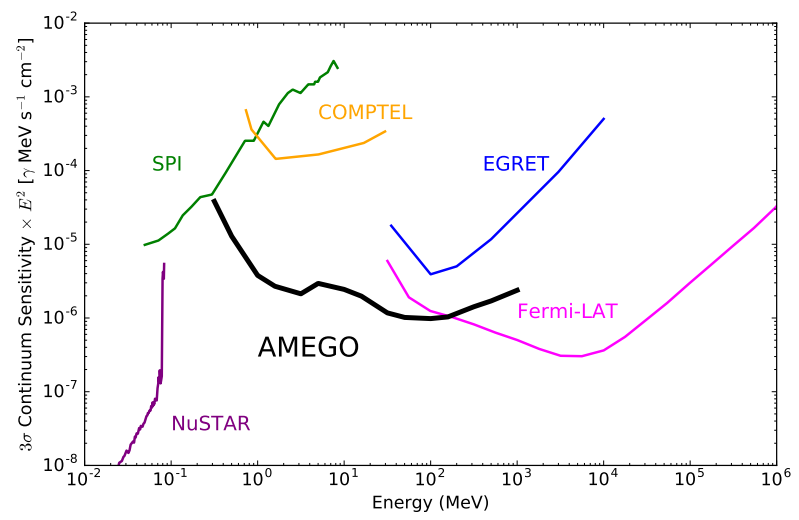

Figure 5: The simulated AMEGO $3 \sigma$ continuum sensitivity with incoming gamma-rays at an incidence angle of $37^{\circ}$. We assumed a 3 -year mission or an exposure of 3 years with a $20 \%$ efficiency (due to field of view and SAA). Both the incidence angle and the efficiency were chosen to partially account for the orbit. For comparison, we show the sensitivity for the Fermi-LAT [13], which assumes the same incidence angle and efficiency but for a 5 year mission. For comparison, we also show the 2 week sensitivity for COMPTEL [14] and EGRET [15] which represents their typical exposure on any point on the sky, both of which assume a $40 \%$ efficiency. If we were to compare AMEGO with a 2 week exposure it would still have a sensitivity x10 better than COMPTEL. NuSTAR [16] and SPI [17] both assume an exposure of $10^{6} \mathrm{~s}$.

We plot the sensitivity of AMEGO as a function of energy (Fig. 5). For comparison, we also show the sensitivities of NuSTAR [16] ${ }^{2}$, INTEGRAL SPI [17] ${ }^{3}$, COMPTEL [14], EGRET [15] and Fermi-LAT [13]. Although comparing instruments is inherently challenging given the different observing strategies and fields-of-view, it has become customary to do so. In the energy band of $\sim 200 \mathrm{keV}$ to $\sim 100 \mathrm{MeV}$ AMEGO is at least a order of magnitude more sensitive than the previous telescopes. This is due mostly to the increased $\mathrm{A}_{\mathrm{eff}}$ and better ARM.

\footnotetext{
${ }^{2}$ https://heasarc.gsfc.nasa.gov/docs/nustar/NuSTAR_observatory_guide-v1.0.pdf

${ }^{3}$ http://integral.esac.esa.int/AO14/AO14_SPI_ObsMan.pdf
} 


\section{Polarization}

In the Compton regime, AMEGO is sensitive to polarization due to the fact that photons preferentially scatter perpendicular to the polarization plane as described by the Klein-Nishina cross section (e.g. [18]),

$$
\frac{d \sigma}{d \Omega}=\frac{r_{0}^{2}}{2} \frac{E_{1}^{2}}{E_{0}^{2}}\left(\frac{E_{0}}{E_{1}}+\frac{E_{1}}{E_{0}}-2 \sin ^{2} \theta \cos ^{2} \eta\right)
$$

where $E_{0}$ and $E_{1}$ are the photon energies before and after scattering, respectively, $\theta$ is the scattering angle, $\eta$ is the angle between the polarization direction of the incident photon and the scattering plane, and $r_{0}$ is the classical electron radius.

In order to evaluate the polarization sensitivity of AMEGO, we performed a set of simulations with $100 \%$ linearly polarized photons. We then scaled the azimuthal scattering angle distribution of the triggered events in the plane normal to the incident direction to the corresponding distribution for unpolarized photons in order to account for azimuthal asymmetries in the detection efficiency. By fitting a sine function to the resulting distribution we determined the relative amplitude of the azimuthal modulation. This amplitude for a $100 \%$ polarized beam is

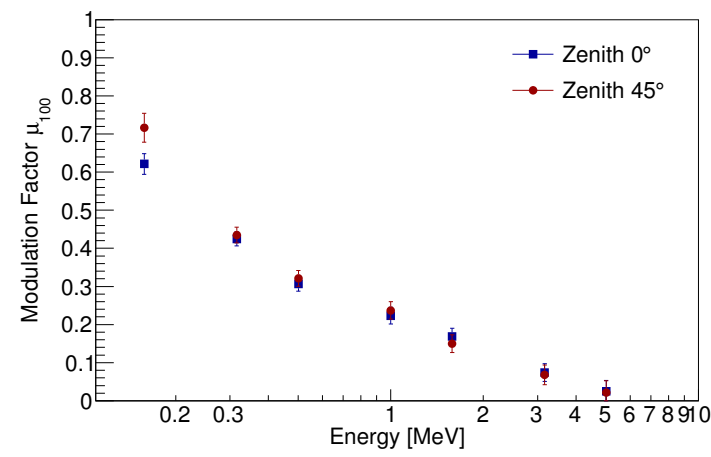

Figure 6: Modulation factor of AMEGO for two different incident zenith angles. called the modulation factor, $\mu_{100}$, and is an important measure of the polarization sensitivity of the instrument. The result is shown in Fig. 6 as a function of energy for two different incident zenith angles.

Given the effective area and background rates described in Sections 4 and 5.1, we estimate the minimum detectable polarization at the $99 \%$ confidence level:

$$
\mathrm{MDP}=\frac{4.29}{\mu_{100} R_{S}} \sqrt{\frac{R_{S}+R_{B G}}{T_{\mathrm{obs}}}},
$$

where $R_{S}$ and $R_{B G}$ are the signal and background event rate from a given source, respectively, and $T_{\mathrm{obs}}$ is the observation time. In one week, assuming that the source is in the field of view for $10 \%$ of the time, AMEGO reaches an MDP of $5 \%(12 \%)$ in the $0.5-1 \mathrm{MeV}(1-2 \mathrm{MeV})$ energy range.

\section{Summary}

We have performed detailed Monte Carlo simulations of the AMEGO detectors using the MEGAlib toolkit to parameterize the instrument. We have found that AMEGO has a sensitivity approaching a factor of 20-50 better than previous gamma-ray observatories in addition to a larger effective area and better angular resolution. We are also continually optimizing the observatory design and developing new tools to better meet the requirements of our core science. AMEGO opens up a new regime of unprecedented sensitivity in the gamma-ray energy range from $200 \mathrm{keV}$ to $>10 \mathrm{GeV}$. 


\section{References}

[1] A. Moiseev, All-Sky Medium Energy Gamma-ray Observatory (AMEGO), in 35th International Cosmic Ray Conference, vol. 35 of International Cosmic Ray Conference, p. 8, July, 2017.

[2] J. Racusin and A. Lien, AMEGO: Transients and Multi-Messenger Sources, in 35th International Cosmic Ray Conference, vol. 35 of International Cosmic Ray Conference, p. 8, July, 2017.

[3] J. S. Perkins, M. Ajello, L. Marcotulli, E. Meyer, V. A. Paliya and T. Venters, All-Sky Medium Energy Gamma-ray Observatory (AMEGO), in 35th International Cosmic Ray Conference, vol. 35 of International Cosmic Ray Conference, p. 8, July, 2017.

[4] R. Caputo, M. Meyer and M. A. Sánchez-Conde, AMEGO: Dark Matter, in 35th International Cosmic Ray Conference, vol. 35 of International Cosmic Ray Conference, p. 8, July, 2017.

[5] A. Zoglauer, R. Andritschke and F. Schopper, MEGAlib The Medium Energy Gamma-ray Astronomy Library, New A Rev. 50 (Oct., 2006) 629-632.

[6] A. Zoglauer, G. Weidenspointner, M. Galloway, S. Boggs and C. Wunderer, "Cosima: The cosmic simulator of megalib." 10.1109/NSSMIC.2009.5402128.

[7] A. C. Zoglauer, First light for the next generation of Compton and pair telescopes : Development of new techniques for the data analysis of combined Compton and pair telescopes and their application to the MEGA prototype, Ph.D. thesis, Garching: Max-Planck-Institut für Extraterrestrische Physik, 2006, MPE Report, No. 289, 2006.

[8] D. E. Gruber et al., The spectrum of diffuse cosmic hard x-rays measured with heao-1, Astrophys. J. 520 (1999) 124, [astro-ph/9903492].

[9] FERMI-LAT collaboration, F. Acero et al., Development of the Model of Galactic Interstellar Emission for Standard Point-Source Analysis of Fermi Large Area Telescope Data, Astrophys. J. Suppl. 223 (2016) 26, [1602.07246].

[10] G. Weidenspointner et al., The COMPTEL instrumental line background, A\&A 368 (Mar., 2001) 347-368, [astro-ph/0012332].

[11] A. W. Strong, I. V. Moskalenko and O. Reimer, Diffuse Continuum Gamma Rays from the Galaxy, ApJ 537 (July, 2000) 763-784, [astro-ph/9811296].

[12] FERMI-LAT collaboration, F. Acero et al., Development of the Model of Galactic Interstellar Emission for Standard Point-source Analysis of Fermi Large Area Telescope Data, ApJS 223 (Apr., 2016) 26, [1602.07246].

[13] FERMi-LAT collaboration, W. B. Atwood et al., The Large Area Telescope on the Fermi Gamma-Ray Space Telescope Mission, ApJ 697 (June, 2009) 1071-1102, [0902 . 1089 ].

[14] COMPTEL collaboration, V. Schoenfelder et al., Instrument description and performance of the Imaging Gamma-Ray Telescope COMPTEL aboard the Compton Gamma-Ray Observatory, Astrophys. J. Suppl. 86 (1993) 657.

[15] EGRET collaboration, D. J. Thompson et al., Calibration of the Energetic Gamma-Ray Experiment Telescope (EGRET) for the Compton Gamma-Ray Observatory, ApJS 86 (June, 1993) 629-656.

[16] NuSTAR collaboration, F. A. Harrison et al., The Nuclear Spectroscopic Telescope Array (NuSTAR) High-energy X-Ray Mission, ApJ 770 (June, 2013) 103, [1301. 7307].

[17] C. Winkler, The INTEGRAL mission, Experimental Astronomy 6 (Dec., 1995) 71-76.

[18] R. D. Evans, The Atomic Nucleus. McGraw-Hill, New York, 1955. 